\title{
Image-injected frameless stereotactic approach to the anterior craniovertebral junction
}

\section{Timothy Ryken, M.D., Terrence Julien, M.D., Bruce Frankel, M.D., Greg Canute, M.D., John Haller, Ph.D., and Arthur Rosenbaum, M.D.}

Division of Neurosurgery and Department of Radiology, University of Iowa Hospitals and Clinics, Iowa City, Iowa; and Departments of Neurosurgery and Radiology, State University of New York Health Science Center, Syracuse, New York.

Transoral odontoidectomy is often performed in the treatment of cervicomedullary junction disease. The operating microscope is frequently used to improve visualization in this narrow field of view. In the setting of complex anatomy or surgical revision the authors hypothesized that combining frameless stereotactic technique with intraoperative microscopy would improve the ability to visualize and identify intraoperative anatomy. In addition they believed that the ability to visualize the targeted region directly in the operating microscope "image injection" would be of particular interest in this setting, provided that sufficient accuracy for use could be obtained in the registration process. The authors assessed the efficacy of this approach in a cadaveric model and obtained sufficient accuracy to warrant use in the operating room. This technique was applied in the surgical management of a 56-year-old woman with rheumatoid arthritis who had undergone a previous decompressive transoral procedure. Subsequently she suffered progressive deterioration and was found to have residual bony compression of the anterior cervicomedullary junction. The authors performed decompressive surgery and obtained satisfactory results by using the image-injected technique, and the patient experienced subsequent clinical improvement. The authors conclude that the image-injected frameless stereotactic technique is of potential benefit, particularly in the narrow window of approach of the transoral odontoidectomy.

Key Words * craniovertebral junction * frameless stereotaxy * computer-assisted surgery * rheumatoid arthritis * cervicomedullary compression

Frameless stereotactic surgical techniques are being rapidly developed and used in many areas of neurosurgery.[1,2,10,11] Systems for both intracranial and spinal use have been developed and continue to undergo refinement and additional development. The main goal of these systems is to provide information to the surgeon concerning the location and relationship of anatomical structures to images obtained preoperatively with this system; the ultimate goal is to provide the surgeon with rapid, accurate, intraoperative localization of anatomy. The instruments used to track intraoperative position can vary from pointed probes to more functional instruments, such as biopsy needles or bipolar forceps, and these 
instruments can be tracked during an operation to provide feedback on the relationship of the instruments to preselected targeted images. Another technique is to substitute the focal point of an operating microscope for the tip of a rigid instrument. In this way, the focal point of the microscope serves as the pointer, and anatomical information is provided based on where the microscope is focused. In addition, once the operating microscope is in use, the preselected target volumes of interest and the preselected surgical plan can be projected into one of the oculars of the microscope head. This technique, which we refer to as "image injection," allows the surgeon to visualize the preselected targets in the surgical field without manipulating additional probes or pointing devices while using the operating microscope. We theorized that this technique would be of particular benefit when performing the transoral approach because of the limited available exposure. It may also be beneficial in surgical revision in which the normally complex anatomy of the craniovertebral junction $(\mathrm{CVJ})$ is further distorted by pathological and surgical changes.

\section{CADAVER STUDY}

Three formalin-fixed cadaveric head and neck preparations were selected for study. To assess our ability to image and reconstruct satisfactorily the craniovertebral junction, six-skin based fiducial markers were placed over the forehead, zygoma, and malar eminence bilaterally, and volumetric axial computerized tomography scans were obtained with 1-mm-slice increments. The images were reconstructed using the Zeiss MKM software system (Thornwood, NY). Three-dimensional reconstruction of the craniovertebral junction was performed for each of the specimens and corresponded well with the preoperatively obtained images. To test the accuracy of the registration process when using this type of fiducial marker placement, one of the cadavers was selected to undergo a surgical procedure. The head and neck preparation was mounted supine in a Mayfield-type headclamp in the operating room. The fiducial markers were registered and yielded a preoperative accuracy of $1.43 \mathrm{~mm}$. The registration process required 6 minutes. A series of landmarks were then selected for targeting, including the midline of the anterior arch of $\mathrm{C}-1$, the tip of the odontoid process, and the junction of the odontoid process with the base of C-2 bilaterally. These targets were dissected and localized using the image-injection technique. In all cases the difference between the virtual and actual targets was between 1 and $2 \mathrm{~mm}$.

\section{CASE REPORT}

History. A 56-year-old right handed woman with a 20-year history of rheumatoid arthritis had originally presented to another surgeon; at the time she was experiencing progressive myelopathic symptoms. Imaging studies had revealed cervicomedullary compression presumably secondary to atlantoaxial instability, and she underwent a posterior fossa decompressive procedure and occiptiocervical fusion. Because she experienced no significant improvement, she underwent decompressive transoral surgery, which was performed several months later. Unfortunately she again failed to improve, and repeated imaging revealed residual bony decompression anteriorly. She was referred to us for a second opinion.

Presentation and Examination. At that time she had complaints of intermittent incontinence and difficulty with fine motion of the hands and ataxia. On neurological examation she was myelopathic, with a 5- left hemiparesis and a 4+ right hemiparesis. Review of her imaging studies including computerized tomography scans and magnetic resonance images of the CVJ, indicated the presence of residual anterior bony compression, which prevented satisfactory decompression (Fig. 1). 


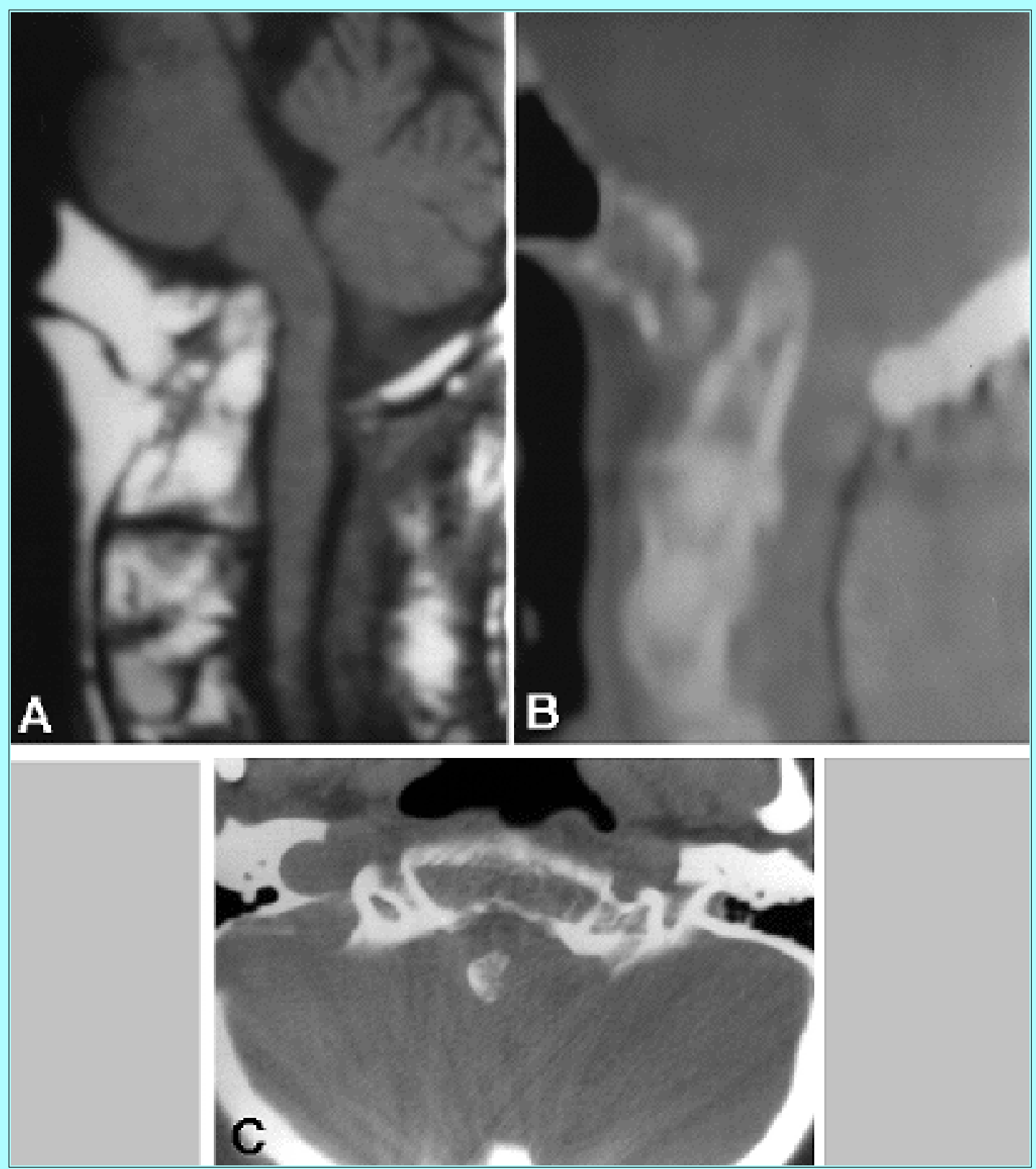

Fig. 1. Preoperative imaging studies. A: A $\mathrm{T}_{1}$-weighted magnetic resonance image of the CVJ. Note the continued anterior compression of the cervicomedullary junction. B and C: Axial computerized tomography scans, reconstructed midline sagittal view (B) and axial view $(\mathrm{C})$, of the $\mathrm{CVJ}$ revealing partial resection of the odontoid process.

Operation. We elected to utilize the Zeiss MKM frameless stereotactic unit to facilitate preoperative planning and intraoperative guidance (Fig. 2). The patient was positioned supine in a Mayfield headrest to allow visualization of a series of eight skin fiducial markers that were placed bifrontally and over the zygomatic arches bilaterally. 

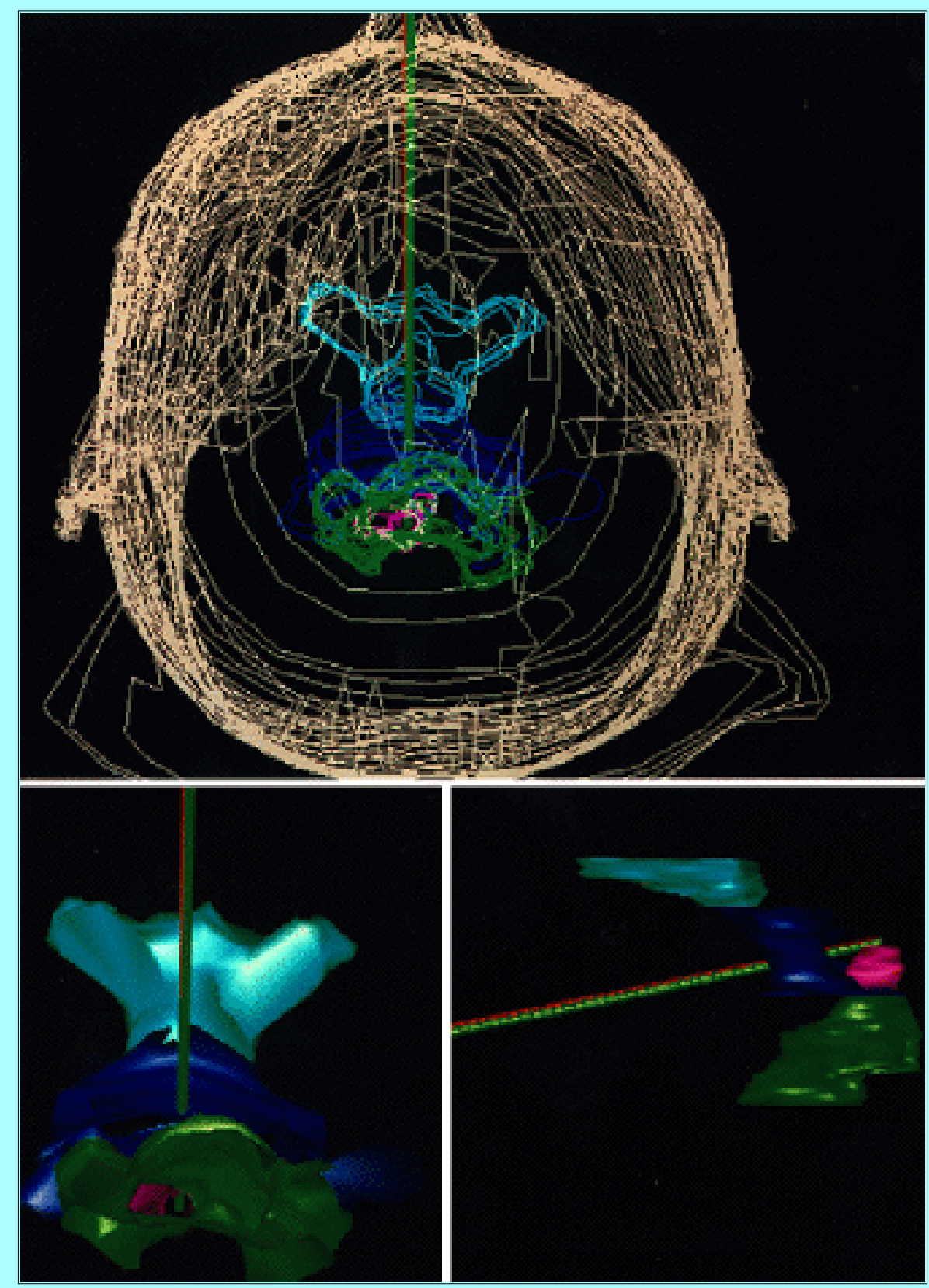

Fig. 2. Preoperative computer-reconstructed views of the CVJ. Upper: Contoured projection of the surgical anatomy viewed in the axial projection. Lower Left: Solid contoured image of the craniocervical structures in axial projection; the distal clivus is depicted in dark blue overlying the residual odontoid in magenta. Lower Right: Lateral projection demonstrating the targeted region (depicted in magenta) and the line of approach to the tip of the residual odontoid process.

Using the Zeiss MKM system the surgeon must be able to focus on the fiducial landmarks to complete the registration process (Fig. 3 left). This was accomplished in 6 minutes, and the preoperative registration accuracy was $1.50 \mathrm{~mm}$. The patient then underwent transoral transpharyngeal approach to the residual ondontoid process. During the decompressive procedure, the image injected into the microscope ocular was used to guide the dissection through the previously formed scar tissue and allow resection of the residual bony compression (Fig. 3 right). 


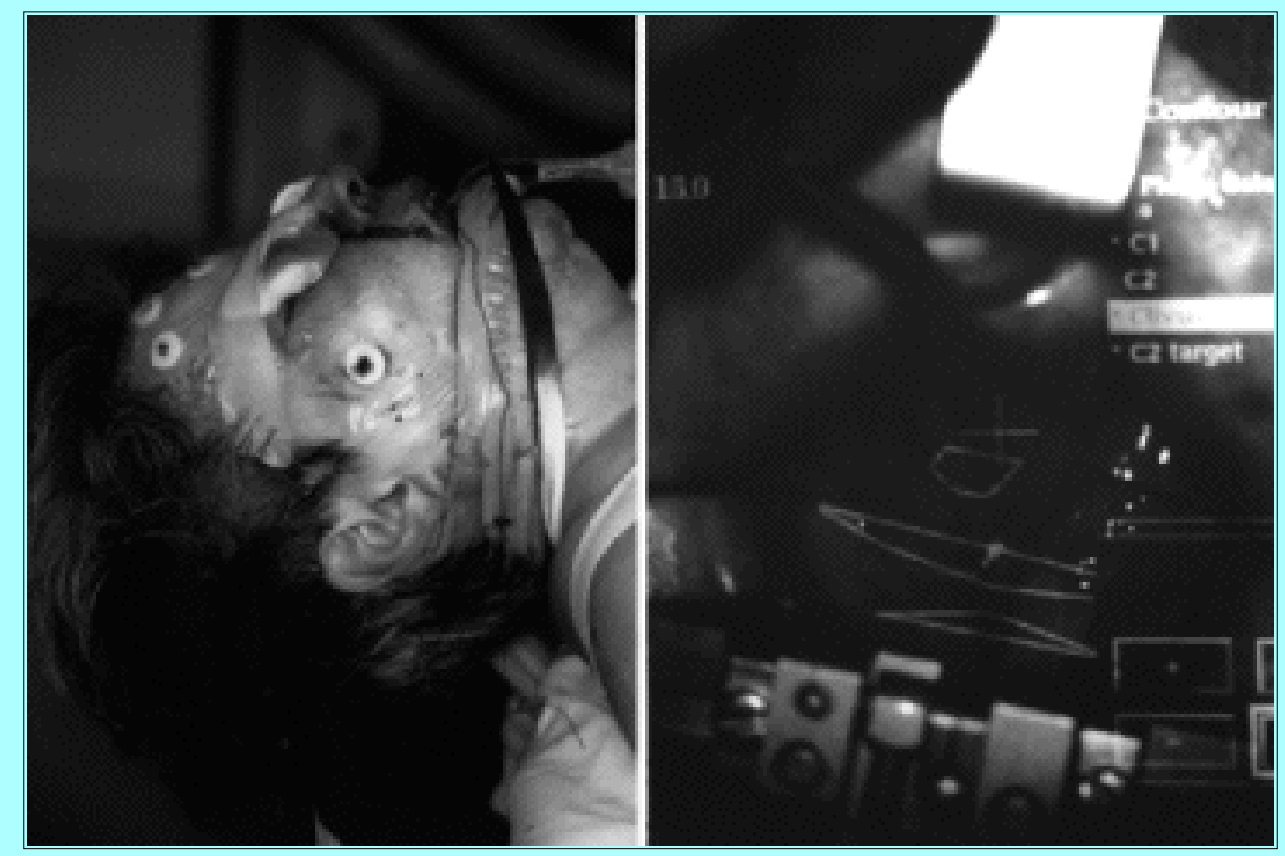

Fig. 3. Positioning and intraoperative photographs showing the image-injected contours and targeting. Left: Note the placement of the fiducial markers for registration. Right: View obtained through the operating microscope indicating the cross-sectional representation of the contoured images at the level of focus.

Postoperative Course. The patient experienced improved strength in the 1st week and at follow-up examination 2.6 years postsurgery has full strength, no difficulty with incontinence, and is fully ambulatory but remains hyperreflexic (Fig. 4). 


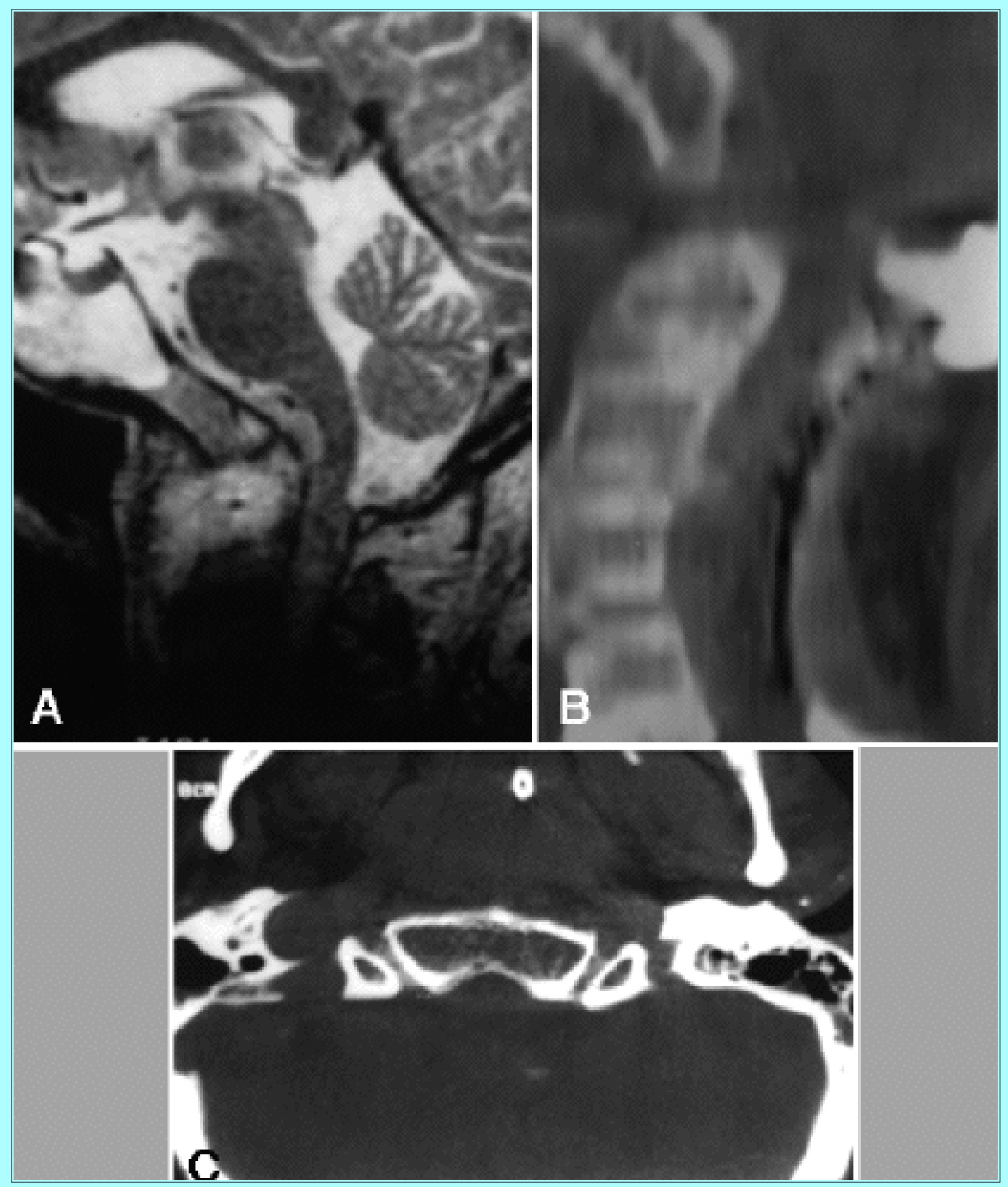

Fig. 4. Postoperative imaging studies. A: Magnetic resonance image of the $\mathrm{CVJ}$ revealing that the anterior cervicomedullary junction has been decompressed. B and C: Axial computerized tomography scans, reconstructed midline sagittal image (B) and axial image (C), of the CVJ confirming resection of the residual odontoid process.

\section{DISCUSSION}

Surgical approaches to the CVJ are among the more challenging of spinal operative procedures.[3-8] The transoral exposure in particular is limited and the anatomy is often distorted secondary to congenital or degenerative anomalies. $[3,7]$ These considerations in the setting of surgical revision prompted the use of the MKM system in this clinical situation.

The application of frameless stereotaxy in the upper cervical spine has been previously described.[10,11] Welch and colleagues[11] described their experience in using an early robotic-arm frameless stereotactic system in 11 patients. They described a maximum discrepancy of $2 \mathrm{~mm}$ between virtual and actual 
images, which corresponds with our observations while using the MKM system. In their series the major problem appeared to be the limited ability to manipulate the mechanical arm. No clinically significant error was described. To minimize motion-induced error, some patients were placed in halo vests preoperatively, which could add to the rate of procedure-related morbidity.

We had two major concerns with the application of the MKM system to transoral odontoidectomy: registration accuracy and intersegmental motion. The use of skin- and surface-based fiducial marker systems as an appropriate way of allowing a stereotactic space to be defined has been challenged.[9] It appears apparent from our limited experience and that of Welch and colleagues that it is possible to obtain accurate registration by using fiducial markers placed on the face. It seems most practical to place these markers over bony landmarks with little soft tissue or redundant skin to minimize motion. The problems associated with intersegmental motion are not so easily overcome. As an example, in our experience, the utility of frameless stereotaxy for posterior atlantoaxial fixation has been limited. In the majority of our cases, significant motion occured between $\mathrm{C}-1$ and $\mathrm{C}-2$ between the time of imaging and surgical positioning. This intersegmental motion can lead to significant errors in surgical planning and in placement of transarticular screws. However, this problem could be overcome if both $\mathrm{C}-1$ and $\mathrm{C}-2$ were registered and tracked simultaneously. This is not possible when using the MKM system. Thus, we concluded that, for the craniocervical structures to be targeted and tracked, they needed to be immobile. Welch and colleagues[11] have addressed this issue by using preoperative halo vest immobilization. In the case of our patient, the presence of a previous occipitocervical fusion allowed us to assume safely that no intersegmental motion had occurred between the time of imaging and operative positioning. In our cadaver dissection, we made a similar assumption because of the stiffness and contraction of the formalin-fixed muscles. Most current frameless stereotactic systems for use in spine procedures require single-segmental registration. Segmental registration requires each rigid body to be registered independently. The major drawbacks are the time spent registering and the inability to track two or more vertebral bodies concurrently, that is, if the two are not assumed to be fused together. Multilevel registration techniques are undergoing development but are not widely used with current frameless systems. The ability to register and track multiple vertebral levels concurrently will constitute a major development and will greatly enhance the practicality of performing these techniques in more routine spine surgery.

The use of frameless guidance systems is not a substitution for good surgical technique, and a thorough understanding of the anatomy is crucial to the efficacy these procedures. Frameless stereotaxy can significantly enhance one's ability to reconstruct visually the three-dimensional (3-D) anatomy, and it allows a thorough inspection of the anatomical images in multiple reconstructed views prior to commencing a procedure. Our experience indicates that the ability to reconstruct the images preoperatively as well as the use of the system in preoperative planning are often of at least equal benefit to the intraoperative guidance. Once the procedure is underway, it serves as a valuable verification for intraoperative localization. Whereas most systems rely on a localizing probe, it is possible conceptually to replace the tip of the probe with the focal point of an operating microscope, which allows the focal point of the microscope to become the point of interest. By building 3-D contour maps of regions of interest prior to initiating the surgical procedure, the cross-sectional representation of the 3-D figure at the level of the focal plane can be projected into the ocular of the microscope. This provides the surgeon with immediate and accurate feedback of the location and relationship of the targeted areas (in our case, the residual odontoid process), and it can allow a more direct and focused approach. Anecdotally, we previously used this same technique in a patient undergoing transoral resection and were skeptical when 
the computer images appeared to demonstrate residual bony compression despite the opinion of the surgeon that adequate decompression had been obtained. Postoperative images revealed that the computer images had been correct, and some mild residual bony elements remained. In addition, the use of the image-injection technique and the elimination of the probe for localization allow both of the surgeon's hands to be used for surgical manipulation while still allowing intraoperative tracking to occur.

\section{CONCLUSIONS}

In conclusion, the development of frameless stereotactic procedures has expanded the capabilities of intraoperative-guidance techniques. The ability to project the targeted regions of interest into the viewing field of the microscope may be a useful technique in a variety of spinal and intracranial procedures. We believe that it may have particular utility in the limited exposure of the transoral approach to the CVJ. However, it is imperative that the surgeon take into account the potential for intersegmental motion and registration error in interpreting the information provided by these systems during operative procedures.

\section{References}

1. Barnett GH, Kormos DW, Steiner CP, et al: Intraoperative localization using an armless, frameless stereotactic wand. Technical note. J Neurosurg 78:510-514, 1993

2. Barnett GH, Kormos DW, Steiner CP, et al: Use of a frameless, armless stereotactic wand for brain tumor localization with two-dimensional and three dimensional neuroimaging. Neurosurgery 33:674-678, 1993

3. Kelly PJ: Commentary on Welch WC, Subach BR, Pollack IF, et al: Frameless stereotactic guidance for surgery of the upper cervical spine. Neurosurgery 40:964, 1997

4. Menezes AH: Anterior approaches to the craniocervical junction. Clin Neurosurg 37:756-769, 1991

5. Menezes AH: Normal and abnormal development of the craniocervical junction, in Hoff JT, Crockard, A, Hayward R (eds): Neurosurgery: The Scientific Basis of Clinical Practice, ed 2. London: Blackwell Scientific, 1992, pp 63-83

6. Menezes AH, Ryken TC: Craniovertebral abnormalities in Down's syndrome. Pediatr Neurosurg 18:24-33, 1992

7. Menezes AH, Ryken TC: Instrumentation of the craniocervical region, in Benzel EC (ed): Spinal Instrumentation. Park Ridge, Ill: American Association of Neurological Surgeons, 1994, pp 47-62

8. Menezes AH, VanGilder JC: Anomalies of the craniovertebral junction, in Youmans JR (ed): Neurological Surgery, ed 3. Philadelphia: WB Saunders, 1990, Vol 2, pp 1359-1420

9. Menezes AH, VanGilder JC: Transoral-transpharyngeal approach to the anterior craniocervical junction. Ten-year experience with 72 patients. J Neurosurg 69:895-903, 1988

10. Pollack IF, Welch W, Jacobs GB, et al: Frameless stereotactic guidance. An intraoperative adjunct in the transoral approach for ventral cervicomedullary junction decompression. Spine 20:216-220, 1995

11. Welch WC, Subach BR, Pollack IF, et al: Frameless stereotactic guidance for surgery of the upper cervical spine. Neurosurgery 40:958-964, 1997 
Manuscript received April 14, 1999.

Accepted in final form May 6, 1999.

Address reprint requests to: Timothy Ryken, M.D., Division of Neurosurgery, University of Iowa Hospitals and Clinics, 200 Hawkins Drive, Iowa City, Iowa 52242. email: timothy-ryken@uiowa.edu. 